\title{
Choroidal Thickness Analysis in Patients with Erectile Dysfunction
}

\author{
Yong-Kyu Kim ${ }^{1}$ (D) Jang Hoon Lee ${ }^{1}$, Sung Pyo Park ${ }^{1}$ (D) Dae Yul Yang ${ }^{2}$ (D) \\ Departments of ${ }^{1}$ Ophthalmology and ${ }^{2}$ Urology, Kangdong Sacred Heart Hospital, Hallym University College of Medicine, Seoul, Korea
}

Purpose: To investigate the morphological features of choroidal vasculature in patients with erectile dysfunction (ED) by analyzing choroidal thickness using optical coherence tomography.

Materials and Methods: We enrolled 39 patients with ED and 19 controls. ED was defined as an erectile function domain score $<26$ on the International Index of Erectile Function (IIEF) questionnaire. Small-choroidal-vessel-layer (SCVL) thickness was calculated by subtracting large-choroidal-vessel-layer (which corresponded to Haller's layer) thickness from total choroidal thickness. Choroidal thickness was compared between the ED and control groups.

Results: SCVL thickness was lesser in the ED group than in the control group (control, $69.8 \pm 24.3 \mu \mathrm{m} v \mathrm{v}$. ED, $55.1 \pm 19.9 \mu \mathrm{m}$; $\mathrm{p}=0.017$ ). Among patients without diabetes, the ED group showed significantly lesser SCVL thickness than did the control group (control, $77.1 \pm 22.7 \mu \mathrm{m}$ vs. ED, $56.5 \pm 20.9 \mu \mathrm{m} ; \mathrm{p}=0.021$ ). However, among patients with diabetes, choroidal thickness showed no significant intergroup difference. Multiple linear regression analysis revealed that spherical equivalent (standardized coefficient $\beta=0.294 ; p=0.019$ ) and the IIEF erectile function score (standardized coefficient $\beta=0.315 ; p=0.012$ ) were significantly associated with SCVL thickness.

Conclusions: SCVL thickness, including the choriocapillaris layer and medium-sized choroidal vascular layer, decreased in proportion to ED severity, suggesting that microvascular changes in choroidal vessels may occur before specific ocular diseases in patients with ED.

Keywords: Choroid; Erectile dysfunction; Tomography, optical coherence

This is an Open Access article distributed under the terms of the Creative Commons Attribution Non-Commercial License (http://creativecommons.org/licenses/by-nc/4.0) which permits unrestricted non-commercial use, distribution, and reproduction in any medium, provided the original work is properly cited.

\section{INTRODUCTION}

Erectile dysfunction (ED) is the inability to achieve or maintain a penile erection sufficient for satisfactory sexual performance [1]. The causes of ED are broadly divided into organic and psychological, but most of them are thought to be caused by organic components [1]. ED might be thought of as a natural consequence of the aging process; however, ED may occur as a result of specific illness. In fact, accumulated evidence suggests

Received: May 11, 2018 Revised: Jul 31, 2018 Accepted: Aug 7, 2018 Published online Nov 5, 2018

Correspondence to: Dae Yul Yang iD https://orcid.org/0000-0002-7331-302X

Department of Urology, Kangdong Sacred Heart Hospital, Hallym University College of Medicine, 150 Seongan-ro, Gangdong-gu, Seoul 05355, Korea.

Tel: +82-2-2224-2291, Fax: +82-2-2224-2769, E-mail: yang1408a@daum.net

Correspondence to: Sung Pyo Park iD https://orcid.org/0000-0002-6978-0311

Department of Ophthalmology, Kangdong Sacred Heart Hospital, Hallym University College of Medicine, 150 Seongan-ro, Gangdong-gu, Seoul 05355, Korea.

Tel: +82-2-2224-2274, Fax: +82-2-470-2088, E-mail: eyepyo@gmail.com 
that ED may be another sign of cardiovascular disease [2-4].

According to the 'artery size' hypothesis, the clinical signs of atherosclerosis are likely to present earlier in small-sized arteries, such as the penile artery, than in relatively large-sized arteries, such as the coronary artery [5,6]. Applying the same logic, it is possible to detect vascular changes earlier in the smaller retinal or choroidal vessels of the eye than in the relatively larger penile artery [7].

The presence of diabetes mellitus increases the risk of ED [8,9]. Furthermore, a significant association has been shown between the severity of diabetic retinopathy and ED, suggesting a common microvascular compromise between the two conditions [10]. In patients with type 2 diabetes, narrower retinal arteriolar and wider venular diameters were independently associated with an increased risk of self-reported ED [11]. However, studies on the relationship between the status of the choroidal vessel and the severity of ED are scarce.

The choroid, like the corpus cavernosum, is a tissue with abundant vascularity [12], and phosphodiesterase type 5 (PDE5), which is associated with penile detumescence, is also found in the retinal and choroidal vasculature [13]. Thus, we hypothesized that there might be significant changes in the choroidal vascular structure of patients with ED. In this study, we investigated the morphological features of choroidal vasculature in patients with ED by analyzing choroidal thickness using optical coherence tomography (OCT).

\section{MATERIALS AND METHODS}

\section{Participants}

Between April 1, 2016 and December 31, 2016, patients who visited the urology clinic for the evaluation of ED were recruited for this study. Simultaneously, patients who visited the ophthalmology clinic for diabetic retinopathy screening, cataract evaluation, and symptoms of vitreous floaters or dry eye were also enrolled for the study.

\section{Ethics statement}

The study protocol was reviewed and approved by the institutional review board of Kangdong Sacred Heart Hospital (Seoul, Korea; IRB No. 2016-01-012). All study conduct adhered to the tenets of the Declaration of Helsinki, and written informed consent was ob- tained from all study participants after explanation of the nature and possible consequences of the study.

\section{Inclusion and exclusion criteria}

The study (ED) group consisted of male patients aged 20 to 70 years with ED, which was defined as an erectile function domain score of less than 26 on the International Index of Erectile Function (IIEF) questionnaire. Most of the patients in the ED group were recruited from the urology clinic; however, those who enrolled at the ophthalmology clinic and scored less than 26 on the IIEF questionnaire were also included in the ED group. The control group consisted of male patients aged 20 to 70 years who visited the ophthalmology clinic for one of the following: diabetic retinopathy screening, cataract evaluation, or symptoms of vitreous floaters or dry eye. Patients in the control group had an IIEF erectile function score greater than or equal to 26. The common exclusion criteria for both the ED and control groups were as follows: 1) previous history of PDE5 inhibitor intake within 3 months of study participation; 2) any diabetic retinopathy (e.g., microaneurysms, retinal hemorrhages, or hard exudates) on fundus examination; 3) retinal or choroidal disorders that might influence choroidal thickness, such as uveitis, central serous chorioretinopathy, or retinal vein occlusions; and 4) intraocular surgery, except for uncomplicated cataract surgery.

\section{International Index of Erectile Function questionnaire}

The IIEF is a 15-item self-administered questionnaire that was developed for assessing erectile function. It is a brief, reliable, and valid questionnaire containing questions in five domains: erectile function, orgasmic function, sexual desire, intercourse satisfaction, and overall sexual satisfaction [14]. The erectile function domain is calculated as the sum of the scores of questions 1 through 5 and question 15 . For a 0.5 prevalence rate of $\mathrm{ED}$, the cutoff point for $\mathrm{ED}$ was 25 , with men scoring less than or equal to 25 being classified as having ED [15]. The erectile function domain is known to be a good diagnostic tool, not only in distinguishing the presence or absence of $\mathrm{ED}$, but also in classifying levels of ED severity [15]. 


\section{Ophthalmologic examinations and choroidal thickness measurements}

Patients underwent routine ophthalmologic examinations, including slit-lamp examination, fundoscopy, and OCT. Best-corrected visual acuities were measured using the Snellen chart and converted to logarithm of minimal angle resolution for statistical evaluation. Refractive errors were evaluated using an auto keratorefractometer (KR-8900; Topcon Corporation, Tokyo, Japan). Patients underwent indirect ophthalmoscopic examination and OCT (Spectralis OCT; Heidelberg Engineering, Heidelberg, Germany) to rule out any retinal disorders. Choroidal thickness and choroidal sublayer thickness were measured according to methods described in a previous study [16,17]. In brief, choroidal thickness was measured using horizontal OCT scan centered on foveal center, which was taken by enhanced depth imaging mode with 100-line scan images averaged. Choroidal thickness was measured perpendicularly from the outer border of the hyperreflective retinal pigment epithelium to the inner sclera at three locations: at the fovea, $750 \mu \mathrm{m}$ temporal to the fovea, and $750 \mu \mathrm{m}$ nasal to the fovea. At the same locations, large choroidal vessels measuring $100 \mu \mathrm{m}$ or more, located close to the choroid-sclera border, and closest to the choroidal thickness measurement locations were selected (Fig. 1, blue asterisks). Perpendicular lines from the innermost point of the large choroidal vessels were drawn (Fig. 1, green dashed lines), which intersected the choroidal thickness measurement lines (Fig. 1, yellow dashed arrows). Large-choroidal-vessel-layer (LCVL) thickness, which corresponded to Haller's layer, was defined as the distance between the intersection point on the choroidal thickness measurement line and the inner border of the sclera (orange arrows). Smallchoroidal-vessel-layer (SCVL) thickness, which corresponded to the complex formed by the choriocapillaris layer and Sattler's layer, was defined as the difference between total choroidal thickness and LCVL thickness (Fig. 1, red arrows). The ratio of LCVL thickness to total choroidal thickness was also calculated. Two observers (YKK, JHL) independently measured choroidal thickness masked to the patient information, and data from the left eyes of the patients were used for evaluation. If there was more than a $30-\mu \mathrm{m}$ difference between each measurement exceeding the previously reported value of interobserver coefficients of repeatability [18], the choroidal thickness measurement was re-reviewed and reassessed. The average values of the two observers were used in analyses. We averaged the choroidal thickness measurements at three different locations, i.e., subfoveal, temporal, and nasal locations, and this average value was used for analysis.

\section{Statistical analyses}

We compared average choroidal thickness and choroidal sublayer thickness between the ED and control groups by using Student t-test. In a previous study, subfoveal choroidal thickness was significantly lesser in patients with proliferative diabetic retinopathy or diabetic macular edema than in healthy controls. Although there were no significant differences in choroidal thickness between patients with nonproliferative diabetic retinopathy and healthy controls [17], we assumed that the presence of diabetes even without any diabetic retinopathy might also influence choroidal thickness. Thus, we compared the average choroidal thickness between patients with and without diabetes. We also divided the patients into subgroups according to the presence or absence of diabetes, and compared the choroidal thickness and its sublayer thickness between patients with ED and the controls in each subgroup by using the Mann-Whitney U-test. We performed multiple linear regression analysis for factors associated with SCVL thickness by using clinical fac-

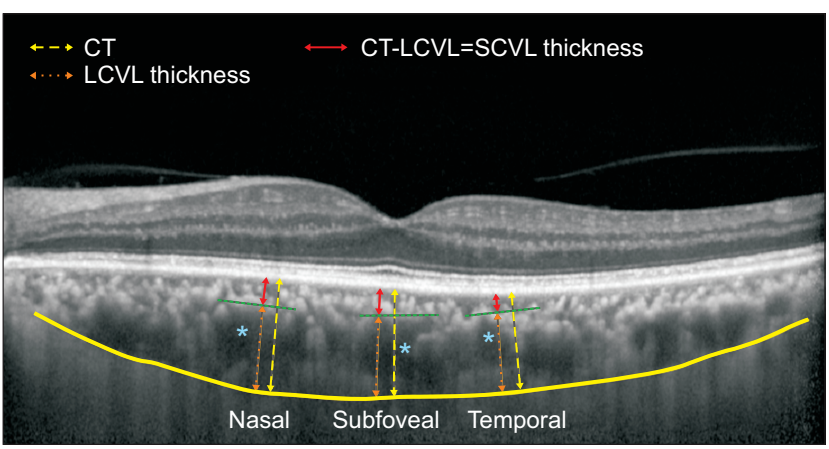

Fig. 1. Choroidal thickness (CT) measurements. CT (yellow dashed arrows) was measured perpendicularly from the outer border of the retinal pigment epithelium to the inner sclera (yellow line) at three locations: at the fovea, $750 \mu \mathrm{m}$ temporal to the fovea, and $750 \mu \mathrm{m}$ nasal to the fovea. Blue asterisks represent the large choroidal vessel located close to the CT measurement locations. Large-choroidalvessel-layer (LCVL) thickness (orange dashed arrows) was measured from the innermost point of the large choroidal vessel (green dashed lines) to the inner sclera. Small-choroidal-vessellayer (SCVL) thickness (red arrows) was calculated by subtracting LCVL thickness from total CT. We averaged the CT measured at three locations, i.e., subfoveal, temporal, and nasal locations, and used this average value for analysis. 
tors such as age, refractive errors which is known to be associated with choroidal thickness, hemoglobin A1c level and duration of diabetes, which represented the severity of diabetes, anti-hypertensive drug use which may have vasodilatory effect and IIEF erectile function domain scores representing the severity of ED by using a stepwise regression approach. Statistical analyses were performed using statistical software (Stata ver. 14.0; Stata Corp., College Station, TX, USA) and statistical significance was defined as $\mathrm{p}<0.05$.

\section{RESULTS}

In this study, we enrolled 39 patients with ED into the ED group, and 19 patients without ED into the control group. Demographics, clinical characteristics, and choroidal thickness were compared between the two groups (Table 1). Interobserver reproducibility of the choroidal thickness measurements was excellent, with intraclass correlation coefficients ' $(2,1)$ ' values were

Table 1. Comparison of demographics and CT between patients in the control and erectile dysfunction groups

\begin{tabular}{lccc}
\hline \multicolumn{1}{c}{ Variable } & $\begin{array}{c}\text { Control } \\
\text { ( } \mathrm{n}=19 \text { eyes) }\end{array}$ & $\begin{array}{c}\text { Erectile } \\
\text { dysfunction } \\
\text { ( } \mathrm{n=39} \text { eyes) }\end{array}$ & p-value ${ }^{\mathrm{a}}$ \\
\hline Age $(\mathrm{y})$ & $54.4 \pm 6.0$ & $56.9 \pm 5.9$ & 0.140 \\
Diabetes mellitus & $7(36.8)$ & $17(43.6)$ & 0.624 \\
Hypertension & $4(21.1)$ & $11(28.2)$ & 0.752 \\
Anti-hypertensive medications & & & \\
CCB & $3(15.8)$ & $5(12.8)$ & $>0.999$ \\
ARB/ACEi & $2(10.5)$ & $4(10.3)$ & $>0.999$ \\
Beta blocker & 0 & $3(7.7)$ & 0.544 \\
HbA1c (\%) & $6.2 \pm 1.0$ & $6.1 \pm 1.1$ & 0.895 \\
BCVA (LogMAR) & $0.03 \pm 0.04$ & $0.03 \pm 0.07$ & 0.812 \\
Refractive errors (SE, diopters) & $-0.9 \pm 1.6$ & $-0.3 \pm 1.9$ & 0.289 \\
CT & & & \\
Total CT ( $\mu$ m) & $297.2 \pm 103.0$ & $274.1 \pm 104.4$ & 0.431 \\
LCVL thickness $(\mu \mathrm{m})$ & $227.4 \pm 86.7$ & $219.0 \pm 92.2$ & 0.743 \\
SCVL thickness $(\mu \mathrm{m})$ & $69.8 \pm 24.3$ & $55.1 \pm 19.9$ & 0.017 \\
Ratio of LCVL to CT & $75.9 \pm 5.8$ & $79.0 \pm 6.1$ & 0.070 \\
IIEF erectile function & $27.3 \pm 1.3$ & $13.4 \pm 6.8$ & $<0.001$ \\
\hline
\end{tabular}

Values are presented as mean \pm standard deviation or number (\%). CT: choroidal thickness, CCB: calcium channel blocker, ARB/ACEi: angiotensin II receptor blocker/angiotensin converting enzyme inhibitor, HbA1c: hemoglobin A1c, BCVA: best-corrected visual acuity, LogMAR: logarithm of the minimum angle of resolution, SE: spherical equivalent, LCVL: large-choroidal-vessel-layer, SCVL: small-choroidalvessel-layer, IIEF: International Index of Erectile Function.

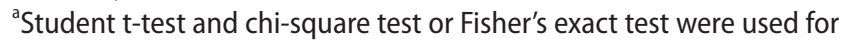
continuous and categorical variables, respectively.
0.990, 0.990, 0.992 for subfoveal, temporal and nasal total choroidal thickness, respectively and 0.838, 0.871, 0.850 for subfoveal, temporal and nasal LCVL thickness, respectively. No significant intergroup differences were observed in terms of age, underlying diabetes or hypertension, use of anti-hypertensive drugs, visual acuity, and degree of refractive errors. No significant intergroup difference was observed in total choroidal thickness or LCVL thickness. However, SCVL thickness was significantly lesser in the ED group than in the control group (control, $69.8 \pm 24.3 \mu \mathrm{m} v$ s. ED, 55.1 \pm 19.9 $\mu \mathrm{m} ; \mathrm{p}=0.017)$.

The comparison between patients with and without diabetes revealed no significant differences in terms of age, visual acuity, degree of refractive errors, and the IIEF erectile function score. Although no significant intergroup differences were observed in total choroidal thickness, LCVL or SCVL thickness, patients with diabetes showed significantly larger ratio of LCVL to total choroidal thickness (no diabetes, $76.3 \pm 6.2$ vs. dia-

Table 2. Comparison of demographics and CT between patients with and without diabetes mellitus

\begin{tabular}{lccc}
\hline \multicolumn{1}{c}{ Variable } & $\begin{array}{c}\text { Without } \\
\text { diabetes } \\
\text { mellitus } \\
\text { (n=34 eyes) }\end{array}$ & $\begin{array}{c}\text { With } \\
\text { diabetes } \\
\text { mellitus }\end{array}$ & p-value ${ }^{\mathrm{a}}$ (24 eyes) \\
\hline Age (y) & $55.7 \pm 6.7$ & $56.8 \pm 4.8$ & 0.498 \\
Hypertension & $6(17.6)$ & $9(37.5)$ & 0.089 \\
Anti-hypertensive medications & & & \\
CCB & $3(8.8)$ & $5(20.8)$ & 0.255 \\
ARB/ACEi & $3(8.8)$ & $3(12.5)$ & 0.684 \\
Beta blocker & $1(2.9)$ & $2(8.3)$ & 0.564 \\
HbA1c (\%) & $5.5 \pm 0.5$ & $7.1 \pm 0.9$ & $<0.001$ \\
BCVA (LogMAR) & $0.02 \pm 0.05$ & $0.04 \pm 0.07$ & 0.260 \\
Refractive errors (SE, diopters) & $-0.5 \pm 1.3$ & $-0.5 \pm 2.4$ & 0.889 \\
CT & & & \\
Total CT ( $\mu$ m) & $277.3 \pm 99.8$ & $287.9 \pm 110.7$ & 0.704 \\
LCVL thickness $(\mu \mathrm{m})$ & $213.5 \pm 85.6$ & $233.4 \pm 96.0$ & 0.409 \\
SCVL thickness $(\mu \mathrm{m})$ & $63.8 \pm 23.5$ & $54.5 \pm 19.8$ & 0.119 \\
Ratio of LCVL to CT & $76.3 \pm 6.2$ & $80.3 \pm 5.3$ & 0.012 \\
IIEF erectile function & $19.3 \pm 7.2$ & $16.0 \pm 10.3$ & 0.185 \\
\hline
\end{tabular}

Values are presented as mean \pm standard deviation or number (\%). $\mathrm{CT}$ : choroidal thickness, CCB: calcium channel blocker, ARB/ACEi: angiotensin II receptor blocker/angiotensin converting enzyme inhibitor, HbA1c: hemoglobin A1c, BCVA: best-corrected visual acuity, LogMAR: logarithm of the minimum angle of resolution, SE: spherical equivalent, LCVL: large-choroidal-vessel-layer, SCVL: small-choroidalvessel-layer, IIEF: International Index of Erectile Function.

${ }^{a}$ Student t-test and chi-square test or Fisher's exact test were used for continuous and categorical variables, respectively. 
Table 3. Comparison of demographics and CT between patients in the control and ED groups according to the presence or absence of DM

\begin{tabular}{|c|c|c|c|c|c|c|}
\hline \multirow[b]{2}{*}{ Variable } & \multicolumn{3}{|c|}{$\begin{array}{l}\text { Without DM } \\
\quad(n=34)\end{array}$} & \multicolumn{3}{|c|}{$\begin{array}{l}\text { With DM } \\
(n=24)\end{array}$} \\
\hline & $\begin{array}{c}\text { Group A } \\
(n=12)\end{array}$ & $\begin{array}{c}\text { Group B } \\
(n=22)\end{array}$ & $\begin{array}{c}\text { p-value } \\
\text { (Group A vs. } \\
\text { Group B) }\end{array}$ & $\begin{array}{c}\text { Group C } \\
(n=7)\end{array}$ & $\begin{array}{c}\text { Group D } \\
(n=17)\end{array}$ & $\begin{array}{l}\text { p-value }{ }^{\mathrm{a}} \\
\text { (Group C vs. } \\
\text { Group D) }\end{array}$ \\
\hline Age $(y)$ & $53.8 \pm 6.6$ & $56.6 \pm 6.7$ & 0.276 & $55.4 \pm 4.8$ & $57.3 \pm 4.7$ & 0.418 \\
\hline DM & 0 & 0 & - & $7(100)$ & $17(100)$ & - \\
\hline Hypertension & $1(8.3)$ & $5(22.7)$ & 0.389 & $3(42.9)$ & $6(35.3)$ & $>0.999$ \\
\hline \multicolumn{7}{|l|}{ Anti-hypertensive medications } \\
\hline CCB & 0 & $3(13.6)$ & 0.537 & $3(42.9)$ & $2(11.8)$ & 0.126 \\
\hline $\mathrm{ARB} / \mathrm{ACEi}$ & $1(8.3)$ & $2(9.1)$ & $>0.999$ & $1(14.3)$ & $2(11.8)$ & $>0.999$ \\
\hline Beta blocker & 0 & $1(4.5)$ & $>0.999$ & 0 & $2(11.8)$ & $>0.999$ \\
\hline $\mathrm{HbA1c}(\%)$ & $5.6 \pm 0.5$ & $5.4 \pm 0.5$ & 0.261 & $7.1 \pm 1.1$ & $7.1 \pm 0.9$ & $>0.999$ \\
\hline DM duration (y) & $\mathrm{N} / \mathrm{A}$ & $\mathrm{N} / \mathrm{A}$ & - & $6.4 \pm 4.1$ & $8.8 \pm 6.0$ & 0.418 \\
\hline BCVA (LogMAR) & $0.02 \pm 0.04$ & $0.02 \pm 0.05$ & 0.631 & $0.03 \pm 0.05$ & $0.04 \pm 0.08$ & 0.804 \\
\hline Refractive errors (SE, diopters) & $-0.8 \pm 1.4$ & $-0.4 \pm 1.3$ & 0.466 & $-1.1 \pm 2.1$ & $-0.2 \pm 2.6$ & 0.534 \\
\hline \multicolumn{7}{|l|}{$\mathrm{CT}$} \\
\hline Total CT $(\mu \mathrm{m})$ & $302.8 \pm 82.5$ & $263.3 \pm 107.3$ & 0.136 & $287.6 \pm 138.5$ & $288.0 \pm 102.1$ & 0.951 \\
\hline LCVL thickness $(\mu \mathrm{m})$ & $225.7 \pm 68.2$ & $206.8 \pm 94.6$ & 0.383 & $230.2 \pm 118.3$ & $234.8 \pm 89.3$ & $>0.999$ \\
\hline SCVL thickness $(\mu \mathrm{m})$ & $77.1 \pm 22.7$ & $56.5 \pm 20.9$ & 0.021 & $57.4 \pm 23.3$ & $53.3 \pm 18.9$ & 0.664 \\
\hline Ratio of LCVL to CT & $74.2 \pm 5.7$ & $77.4 \pm 6.3$ & 0.087 & $78.7 \pm 5.2$ & $81.0 \pm 5.3$ & 0.349 \\
\hline IIEF erectile function & $27.0 \pm 1.1$ & $15.1 \pm 5.2$ & $<0.001$ & $27.9 \pm 1.3$ & $11.1 \pm 8.1$ & $<0.001$ \\
\hline
\end{tabular}

Values are presented as mean \pm standard deviation or number (\%).

CT: choroidal thickness, ED: erectile dysfunction, DM: diabetes mellitus, Group A and Group C: No ED, Group B and Group D: ED, CCB: calcium channel blocker, ARB/ACEi: angiotensin II receptor blocker/angiotensin converting enzyme inhibitor, HbA1c: hemoglobin A1c, BCVA: best-corrected visual acuity, LogMAR: logarithm of the minimum angle of resolution, SE: spherical equivalent, LCVL: large-choroidal-vessel-layer, SCVL: smallchoroidal-vessel-layer, IIEF: International Index of Erectile Function, N/A: not available.

a Mann-Whitney U-test and chi-square test or Fisher's exact test were used for continuous and categorical variables, respectively.

betes, $80.3 \pm 5.3 ; \mathrm{p}=0.012$; Table 2).

Among patients without diabetes, the ED group showed significantly lesser SCVL thickness (control, $77.1 \pm 22.7 \mu \mathrm{m}$ vs. $\mathrm{ED}, 56.5 \pm 20.9 \mu \mathrm{m} ; \mathrm{p}=0.021)$. In contrast, among patients with diabetes, no significant differences in choroidal thickness were observed between the control and ED groups (Table 3).

On multiple linear regression analysis, only refractive errors (spherical equivalent: standardized coefficient $\beta=0.294 ; p=0.019)$ and the $\Pi \mathrm{EF}$ erectile function score (standardized coefficient $\beta=0.315 ; p=0.012$ ) showed a significant association with SCVL thickness (Table 4).

\section{DISCUSSION}

In this study, we investigated the morphological features of choroidal vasculature in patients with $\mathrm{ED}$ by measuring choroidal thickness using OCT. Our findings revealed no difference in overall choroidal thick-
Table 4. Multiple linear regression analysis for factors associated with small choroidal vessel layer thickness

\begin{tabular}{lcccc}
\hline Variable & $\begin{array}{c}\text { Coefficient } \\
\text { B }\end{array}$ & $\begin{array}{c}\text { Standard Standardized } \\
\text { error }\end{array}$ & p-value ${ }^{\mathrm{a}}$ \\
\hline $\begin{array}{c}\text { Spherical } \\
\text { equivalent }\end{array}$ & 3.558 & 1.471 & 0.294 & 0.019 \\
$\begin{array}{c}\text { IIEF erectile } \\
\text { function score }\end{array}$ & 0.809 & 0.313 & 0.315 & 0.012 \\
\hline
\end{tabular}

${ }^{a}$ Age, spherical equivalent (diopters), hemoglobin A1c, diabetes duration, anti-hypertensive drug use and International Index of Erectile Function (IIEF) erectile function score were entered in the model, and significant factors were selected using stepwise regression.

ness between the ED and control groups. However, the SCVL, which is thought to include the choriocapillaris layer and medium-sized choroidal vessels, was thinner in the ED group than in the control group, and SCVL thickness decreased in proportion to ED severity represented by the IIEF erectile domain function scores. Studies have shown that diabetes is a major risk factor 
for ED [8,19,20], and ED severity is closely related to the severity of diabetic retinopathy [10]. Thus, in this study, we only included those patients with diabetes who did not have any diabetic retinopathy in order to reduce the effect of diabetic microvascular complication on the evaluation of ED and choroidal measurements. We also performed subgroup analysis according to the presence or absence of diabetes. Our results showed that SCVL thinning in the ED group was more evident among patients without diabetes.

The average SCVL thickness of subfoveal, nasal and temporal area was $69.8 \pm 24.3 \mu \mathrm{m}$ in control group and $55.1 \pm 19.9 \mu \mathrm{m}$ in ED group. Branchini et al [16] reported that the combined subfoveal medium-sized choroidal vessel layer and choriocapillaris layer thickness which is corresponded to our measurement of SCVL thickness was $52.9 \pm 20.6 \mu \mathrm{m}$ in healthy eyes, which was lesser than that of our control group. This is thought to be due to the thicker overall choroidal thickness of our subject group and the ratio of LCVL to total choroidal thickness was similar between 0.7 to 0.8. In another study by the same group, SCVL thickness was significantly lesser in patients with proliferative diabetic retinopathy or diabetic macular edema than in healthy controls, but not significantly different between patients with nonproliferative diabetic retinopathy and controls [17]. Our study showed that there were no statistically significant differences in total choroidal thickness, LCVL or SCVL thickness between patients with and without diabetes, however, diabetic patients showed larger ratio of LCVL to total choroidal thickness, suggesting there is a tendency of lesser SCVL thickness in diabetic patients. Our results suggest that microvascular changes in choroidal vasculature may already occur in patients with diabetes but without retinopathy. A recent study using OCT angiography also revealed that choriocapillaris layer alterations were common not only in patients with diabetic retinopathy but also in patients with diabetes but without retinopathy [21].

In subgroup analysis, a significant difference in SCVL thickness was observed between the ED and control groups among patients without diabetes. However, no significant difference in choroidal thickness was observed between the ED and control groups among patients with diabetes. Thinning of the SCVL observed in the current study might appear as part of the microvascular complication manifested by ED or diabetes. However, the choriocapillaris layer accounts for only $5 \%$ to $10 \%$ of the total choroid [22]. Thus, in patients with diabetes whose SCVL has already been compromised, further SCVL damage caused by comorbid ED might be limited, and a larger number of patients will be needed to demonstrate the difference in SCVL thickness between those with and without ED. In contrast, among patients without diabetes, the ED group showed significantly lesser SCVL thickness than did the control group. This suggested that choroidal vasculopathy had already occurred in patients with $\mathrm{ED}$ without obvious ocular disorders, and that SCVL thickness could be a predictive factor of early vasculogenic ED. The results of multiple linear regression showed that only refractive errors and the IIEF erectile domain function score were significantly associated with SCVL thickness, thereby suggesting that SCVL thickness is associated with ED severity.

It is noteworthy that ED can be an early sign of cardiovascular disease as well as a disease in itself. The presence of ED predicted subsequent cardiovascular events to a degree either equal to or greater than that of current smoking or a family history of myocardial infarction [6,23]. Endothelial dysfunction is considered the common mechanism underlying ED and cardiovascular disease [4,6,24,25], and the 'artery size' hypothesis posits that the symptom of atherosclerosis is likely to manifest earlier in arteries with a smaller diameter [5]. Because the present study was cross-sectional, we could not confirm whether patients with thin SCVLs subsequently developed ED or other systemic vascular diseases. However, there are possibilities that vascular compromise could be detected earlier in choroidal vessels with smaller diameters. The blood vessels of the eye are relatively easy to observe and evaluate than the blood vessels in other body parts; this factor needs to be investigated further.

Several studies have investigated the influence of systemic sildenafil on choroidal perfusion and thickness. Both choroidal perfusion and thickness are known to increase following ingesting systemic sildenafil, although it seems have no significant visual effects or even associated with ischemic complication $[26,27]$. It will be noteworthy to evaluate the change in the thickness of each layer of the choroid after administration of sildenafil to fully investigate its effect on the eye.

This study has several limitations. The sample size 
is small, in particular, the number of patients in the group with diabetes but without ED was small because of the nature of this disease. We were not able to consider underlying medical conditions thoroughly, especially psychological factors, which are known to be associated with ED. Although there were no significant differences in terms of proportion of anti-hypertensive drug use between control and ED groups, it is difficult to know how these drugs have affected choroidal thickness. There might also be some errors in choroidal thickness measurement. Although we referred to a previous research method [16,17], we used the average value measured at three macular points to reduce measurement errors. In addition, two observers independently measured choroidal thickness and showed good interobserver reproducibility. In this study, we excluded patients with any retinal or choroidal pathology that might affect choroidal measurements, such as patients with pachychoroid diseases, wherein the choriocapillaris layer might be reduced because of choroidal congestion in larger choroidal vessels [28-30]. The choriocapillaris layer and medium-sized choroidal vessel layer could not be analyzed separately by using the current imaging device, and further studies using recent OCT angiography techniques might be warranted.

\section{CONCLUSIONS}

The thickness of the SCVL including the choriocapillaris layer and medium-sized choroidal vascular layer decreased in proportion to ED severity, and this phenomenon was more evident in patients without diabetes. These findings suggest that microvascular changes in the choroidal vessels may have already occurred in patients with ED without specific ocular diseases and that SCVL thinning may be an early sign of ED.

\section{ACKNOWLEDGEMENTS}

This study was supported by the Kangdong Sacred Heart Hospital Fund (grant no. 2016-03).

\section{Disclosure}

The authors have no potential conflicts of interest to disclose.

\section{Author Contribution}

Conceptualization: Kim YK, Yang DY. Data curation: all authors. Formal analysis: Kim YK. Funding acquisition: Kim YK. Investigation: all authors. Methodology: all authors. Supervision: Kim YK, Park SP, Yang DY. Validation: all authors. Writing (original draft): all authors. Writing (review \& editing): all authors.

\section{REFERENCES}

1. NIH Consensus Conference. Impotence. NIH consensus development panel on impotence. JAMA 1993;270:83-90.

2. Sullivan ME, Thompson CS, Dashwood MR, Khan MA, Jeremy JY, Morgan RJ, et al. Nitric oxide and penile erection: is erectile dysfunction another manifestation of vascular disease? Cardiovasc Res 1999;43:658-65.

3. Shabsigh R, Anastasiadis AG. Erectile dysfunction. Annu Rev Med 2003;54:153-68.

4. Montorsi P, Ravagnani PM, Galli S, Rotatori F, Briganti A, Salonia $\mathrm{A}$, et al. Common grounds for erectile dysfunction and coronary artery disease. Curr Opin Urol 2004;14:361-5.

5. Montorsi P, Ravagnani PM, Galli S, Rotatori F, Briganti A, Salonia A, et al. The artery size hypothesis: a macrovascular link between erectile dysfunction and coronary artery disease. Am J Cardiol 2005;96:19M-23M.

6. Watts GF, Chew KK, Stuckey BG. The erectile-endothelial dysfunction nexus: new opportunities for cardiovascular risk prevention. Nat Clin Pract Cardiovasc Med 2007;4:263-73.

7. Michalinos A, Zogana S, Kotsiomitis E, Mazarakis A, Troupis T. Anatomy of the ophthalmic artery: a review concerning its modern surgical and clinical applications. Anat Res Int 2015. doi: 10.1155/2015/591961.

8. Feldman HA, Goldstein I, Hatzichristou DG, Krane RJ, McKinlay JB. Impotence and its medical and psychosocial correlates: results of the Massachusetts Male Aging Study. J Urol 1994;151:54-61.

9. Malavige LS, Levy JC. Erectile dysfunction in diabetes mellitus. J Sex Med 2009;6:1232-47.

10. Henis O, Shahar Y, Steinvil A, Finn T, Heruti R, Loewenstein A, et al. Erectile dysfunction is associated with severe retinopathy in diabetic men. Urology 2011;77:1133-6.

11. Chew SK, Taouk Y, Xie J, Nicolaou TE, Wang JJ, Wong TY, et al. The relationship of retinal vessel caliber with erectile dysfunction in patients with type 2 diabetes. Invest Ophthalmol Vis Sci 2013;54:7234-9.

12. LeGrand Y, El Hage SG. Physiological optics. Berlin, Heidelberg: Springer-Verlag; 1980;XVII, 340. 
13. Foresta C, Caretta N, Zuccarello D, Poletti A, Biagioli A, Caretti L, et al. Expression of the PDE5 enzyme on human retinal tissue: new aspects of PDE5 inhibitors ocular side effects. Eye (Lond) 2008;22:144-9.

14. Rosen RC, Riley A, Wagner G, Osterloh IH, Kirkpatrick J, Mishra A. The international index of erectile function (IIEF): a multidimensional scale for assessment of erectile dysfunction. Urology 1997;49:822-30.

15. Cappelleri JC, Rosen RC, Smith MD, Mishra A, Osterloh IH. Diagnostic evaluation of the erectile function domain of the International Index of Erectile Function. Urology 1999;54: 346-51.

16. Branchini LA, Adhi M, Regatieri CV, Nandakumar N, Liu JJ, Laver N, et al. Analysis of choroidal morphologic features and vasculature in healthy eyes using spectral-domain optical coherence tomography. Ophthalmology 2013;120:1901-8.

17. Adhi M, Brewer E, Waheed NK, Duker JS. Analysis of morphological features and vascular layers of choroid in diabetic retinopathy using spectral-domain optical coherence tomography. JAMA Ophthalmol 2013;131:1267-74.

18. Rahman W, Chen FK, Yeoh J, Patel P, Tufail A, Da Cruz L. Repeatability of manual subfoveal choroidal thickness measurements in healthy subjects using the technique of enhanced depth imaging optical coherence tomography. Invest Ophthalmol Vis Sci 2011;52:2267-71.

19. Johannes CB, Araujo AB, Feldman HA, Derby CA, Kleinman $\mathrm{KP}, \mathrm{McKinlay}$ JB. Incidence of erectile dysfunction in men 40 to 69 years old: longitudinal results from the Massachusetts male aging study. J Urol 2000;163:460-3.

20. Penson DF, Latini DM, Lubeck DP, Wallace KL, Henning JM, Lue TF. Do impotent men with diabetes have more severe erectile dysfunction and worse quality of life than the general population of impotent patients? Results from the Exploratory Comprehensive Evaluation of Erectile Dysfunction (ExCEED) database. Diabetes Care 2003;26:1093-9.
21. Choi W, Waheed NK, Moult EM, Adhi M, Lee B, De Carlo T, et al. Ultrahigh speed swept source optical coherence tomography angiography of retinal and choriocapillaris alterations in diabetic patients with and without retinopathy. Retina 2017;37:11-21.

22. Ramrattan RS, van der Schaft TL, Mooy CM, de Bruijn WC, Mulder PG, de Jong PT. Morphometric analysis of Bruch's membrane, the choriocapillaris, and the choroid in aging. Invest Ophthalmol Vis Sci 1994;35:2857-64.

23. Thompson IM, Tangen CM, Goodman PJ, Probstfield JL, Moinpour CM, Coltman CA. Erectile dysfunction and subsequent cardiovascular disease. JAMA 2005;294:2996-3002.

24. Maas R, Schwedhelm E, Albsmeier J, Böger RH. The pathophysiology of erectile dysfunction related to endothelial dysfunction and mediators of vascular function. Vasc Med 2002; 7:213-25.

25. Blick C, Ritchie RW, Sullivan ME. Is erectile dysfunction an example of abnormal endothelial function? Curr Vasc Pharmacol 2016;14:163-7.

26. McCulley TJ, Luu JK, Marmor MF, Feuer WJ. Effects of sildenafil citrate (Viagra) on choroidal congestion. Ophthalmologica 2002;216:455-8.

27. Vance SK, Imamura Y, Freund KB. The effects of sildenafil citrate on choroidal thickness as determined by enhanced depth imaging optical coherence tomography. Retina 2011;31:332-5.

28. Pang CE, Freund KB. Pachychoroid neovasculopathy. Retina 2015;35:1-9.

29. Balaratnasingam C, Lee WK, Koizumi H, Dansingani K, Inoue M, Freund KB. Polypoidal choroidal vasculopathy: A distinct disease or manifestation of many? Retina 2016;36:18.

30. Lee WK, Baek J, Dansingani KK, Lee JH, Freund KB. Choroidal morphology in eyes with polypoidal choroidal vasculopathy and normal or subnormal subfoveal choroidal thickness. Retina 2016;36 Suppl 1:S73-82. 